\title{
The Comforting Presence of Relational Agents
}

Timothy Bickmore

Assistant Professor

College of Computer and

Information Science

Northeastern University

360 Huntington Ave, WVH 202

Boston, MA 02115 USA

bickmore@ccs.neu.edu

\section{Daniel Schulman}

College of Computer and

Information Science

Northeastern University

360 Huntington Ave, WVH 202

Boston, MA 02115 USA

schulman@ccs.neu.edu

\begin{abstract}
In this paper we describe an on-going experiment on the calming effects of a relational agent on users following a social bonding interaction. Applications to a range of health care problems are discussed.
\end{abstract}

\section{Keywords}

Comforting; caring; embodied conversational agent; affective computing; social interface; relational agent; emotion.

\section{ACM Classification Keywords}

H5.2 [Information Interfaces and Presentation]: User Interfaces-Evaluation/methodology, Graphical user interfaces, Interaction styles, Natural language, Theory and methods, Voice I/O.

\section{I ntroduction}

Social support has been demonstrated in many studies to have a significant positive impact on health. For example, a number of studies have demonstrated that emotional support, provided in the context of intimate relationships, increases survival rates among people with severe cardiovascular conditions [1].

The effects of all kinds of social support are primarily a function of the perception of support by the one 
receiving it, rather than the perceptions, intentions or actual behavior of the person providing it [18]. Even pets can provide a sense of caring and emotional support, and pet ownership has been found to be correlated with several kinds of beneficial healthrelated effects [19]. One study demonstrated that the mere presence of a companion animal results in significantly lower cardiovascular reactivity (heart rate and blood pressure) following cognitive stressors, compared to the same situation in which the animal was not present [6].

The mere presence of a human friend or significant other has also been shown to reduce cardiovascular reactivity when study subjects were confronted with cognitive stressors [8]. However, other studies have shown opposite results, possibly due to the performance anxiety associated with working through the cognitive stressor tests with someone present.

We are interested in developing interface agents that are capable of conferring the same supportive and calming benefits on users as those described above. Such agents could be used in health care to calm and reassure patients during hospital stays and to provide palliative care. "Virtual doulas" could provide emotional support to women in labor, resulting in the same reductions in cesarean or forceps deliveries and epidural anesthesia found with human doulas [20]. Comforting agents could also provide social support to isolated elders, since some studies have shown that these elders have significantly greater mortality rates than those who have even minimal social contact with friends and family [10].
We hypothesize that in order for computer agents to provide effective comforting they first need to establish social bonds with users. Relational agents are computer agents designed to build and maintain such long-term social emotional relationships with users [3]

In this paper, we discuss preliminary results from a study in which we are investigating whether the mere presence of a relational agent could have a calming effect on users following negative mood and cognitive stress induction procedures in a laboratory setting.

\section{Related Work}

Reeves and Nass were among the first researchers to show that computer agents could affect user attitudes towards them. They demonstrated the ability of computers to increase users' liking of them through the use of flattery, praise of other computers, matching the user in personality, or the use of "in-group" cues [15]. Morkes, Kernal and Nass demonstrated that computer agents that use humor are rated as more likable, competent and cooperative than those that do not [13]

Klein found that computer displays of empathy via text menus were significantly more effective at alleviating users' frustration than identical systems that only allowed users to express their feelings (vent) or ignored their feelings altogether [9]. Brave, et al,

demonstrated that computer agents that manifest "other-oriented" emotions (e.g., empathy) led to more positive ratings of the agent by users, including greater perceived caring and felt support, compared to agents that either did not display any emotions or only displayed "self-oriented" emotions [5]. 
There have also been several studies on the ability of computers to affect users' trust in them. Embodied pedagogical agents, especially those that are highly expressive, have been found to increase students' perceptions of trust: such agents are perceived as helpful, believable, and concerned [11]. Mulken, et al, found that personification of an interface by itself does not appear to be a sufficient condition for raising the trustworthiness of a computer [14]. In an experiment with REA-a life-sized, animated virtual real estate agent-Bickmore showed that the agent's use of social dialogue increased trust in it for extroverts (for introverts it had no effect) [2].

Liu and Picard showed that a wearable computer designed for acquiring health-related information from users was rated as significantly less stressful to use and was more preferred by users when the computer used empathetic language in its interactions with users [12].

Bickmore and Picard demonstrated that a relational agent that used a range of relationship-building behaviors in daily interactions with users over time was able to establish a greater sense of being cared for in users compared to the same agent that did not use these behaviors [4].

Finally, Rickenberg and Reeves demonstrated that an animated character that "monitored" users while they worked led to greater user anxiety compared to the presence of characters that did not appear to monitoring users, and the effect was greatest for users with external orientations (those who thought that other people controlled their success) [16]. However, the characters used in the experiment did not use any relational, comforting or empathetic messages in their interactions with users, nor language of any kind to verbally communicate with users.

\section{Relational Agent}

We are using the relational agent developed for the FitTrack studies in our investigation [3]. This agent appears as an embodied conversational agent that speaks to users using synthesized speech and synchronized nonverbal behavior such as hand gestures, head nods and posture shifts. User utterances are selected from a multiple choice menu (see Figure 1). For the current study we developed a new interaction dialogue script that was approximately 15 minutes in length. The interaction consisted entirely of relationship-building dialogue including social dialogue, meta-relational dialogue, empathy exchanges, humor, and reciprocal self-disclosure exchanges [3].

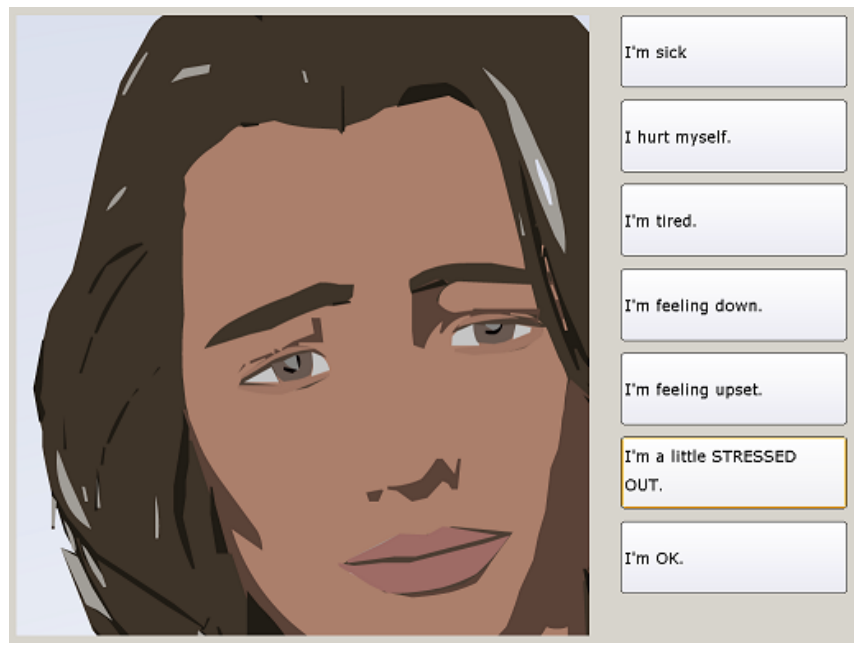

Figure 1. Relational agent interface used in study 


\section{Experimental Methods}

Two experimental designs were implemented in paralle and used on the same subjects in a single session. Both experiments used the same relational agent bonding interaction and rest-period intervention and the same outcome measures. At the start of the session subjects were consented, filled out demographic questionnaires and then conducted the 15-minute relationship-building dialogue with the agent to establish an initial social bond.

In the first experiment (STRESS), a within-subjects test of the ability to calm users following a cognitive stressor was assessed. In this experiment subjects were given either a series of timed math or word problems for a total duration of three minutes (as in [6]). In one condition of this experiment, subjects waited alone during a rest period following a cognitive stress task (STRESS-ALONE). In the second condition of this experiment, subjects waited with the relational agent present on a screen in front of them (STRESSAGENT). The order of presentation of these conditions was completely counterbalanced.

In the second experiment (MOOD), a between-subjects test of the agent's ability to calm users following a negative mood induction was assessed. The induction procedure used a failure manipulation of a "social perception" task in which subjects were asked to identify the emotional states of a series of face images. The task was configured so that subjects' self-scored performance was always reported to be significantly below the mean relative to their peers. Subjects were randomly assigned to either wait alone (MOOD-ALONE) or wait with the agent (MOOD-AGENT) following the induction procedure.
The rest periods following each induction were three minutes long. In the STRESS-AGENT and MOOD-AGENT conditions the agent stated that it was going to wait with the subject, but did not otherwise interact with them. Idle behaviors in the animation engine caused the agent to periodically blink, glance around and change postures. We felt it was important to not have the agent present during the induction procedures to avoid performance anxiety (as observed in [16]).

\section{Apparatus}

Figure 2 shows the experimental setup. During the experiment we recorded subjects' heart rate using a finger-clip blood volume pulse sensor from Thought Technology Ltd.

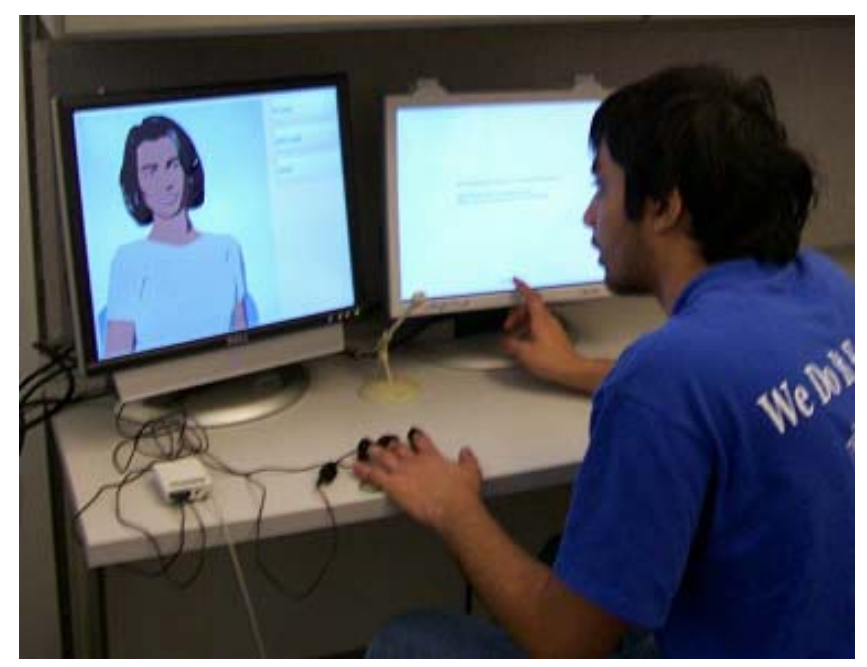

Figure 2. Experimental setup, with relational on left and touch screen induction computer on right. 
We modified the FitTrack relational agent to be controlled via a Wizard-of-Oz apparatus to simulate speech-based input to the agent. Subjects were led to believe the agent could understand their spoken utterances when, in fact, the agent was being controlled by the experimenter (seated in another room) who was listening to them. Relative to menubased input, this apparatus provided a more natural interaction and reduced concerns about motion artifacts in the heart rate sensor. Relative to automated speech recognition, this eliminated concerns about the accuracy of speech recognition.

Subjects were led through the entire experiment, including the administration of questionnaires, by instructions on a touch-screen computer positioned next to the relational agent display.

\section{Measures}

The primary outcome measures used are the Positive and Negative Affect Schedule (PANAS) [17]-a selfreport assessment of mood-administered just before each induction procedure and at the end of each wait period, and heart rate, recorded continuously at $256 \mathrm{~Hz}$ during the study.

\section{Procedure}

After being seated, subjects were connected to the heart rate sensor and given brief instructions, and the experimenter left the room. The relational agent then appeared and conducted the relationship-building dialogue. Subjects then completed three cycles of the following steps: (a) mood assessment; (b) mood induction; and $(\mathrm{c}$ ) rest period (with or without the agent present). A final mood assessment was conducted at the end of the session.

\section{Results}

To date, we have only pilot tested the system on 12 subjects who were recruited from the Northeastern University campus. Currently, there are no significant results to report. However, we have discovered that even though subjects conduct an extended dialogue with the agent, they apparently fail to develop a strong social bond. Subjects rated their relationship with Laura an average score of 2.25 on a scale of 1 ("complete stranger") to 7 ("close friend"), and 4.17 on the Bond subscale of the Working Alliance Inventory [7] (scores to range 1 to 7 ).

\section{Discussion and Future Work}

We plan to revise our protocol to improve the intensity of both the mood induction procedures and the subjectagent social bond and repeat the experiment with more subjects. One strategy to improve bonding is to have subjects conduct multiple interactions with the agent over several days prior to the actual experiment. Other refinements include varying whether the agent performs any social bonding behavior or not, and the inclusion of additional physiological sensors of arousal such as galvanic skin response. This is the first of a series of experiments planned to explore the ability of relational agents to calm and comfort users.

\section{Acknowledgements}

Thanks to Daniel Mauer and Ishraque Nazmi for their help on this project. Thanks also to Jennifer Smith for her many helpful comments on this paper. 


\section{References}

[1] Berkman, L. and Syme, S. Social networks, host resistance, and mortality: A nine year followup study of Alameda County residents. Am J Epidemiol, 109, 1979) 186-204.

[2] Bickmore, T. and Cassell, J., Relational Agents: A Model and Implementation of Building User Trust, $\mathrm{CHI}$ '01, 2001, pp. 396-403.

[3] Bickmore, T. and Picard, R. Establishing and Maintaining Long-Term Human-Computer Relationships. ACM Transactions on Computer Human Interaction, 12, 2, (2005) 293-327.

[4] Bickmore, T. and Picard, R., Towards Caring Machines, CHI'04, 2004.

[5] Brave, S., Nass, C., and Hutchinson, K. Computers that care: investigating the effects of orientation of emotion exhibited by an embodied computer agent. Int J Human-Computer Studies, 62, 2005) 161-178. [6] DeMello, L. The Effect of the Presence of a Companion-Animal on Physiological Changes Following the Termination of Cognitive Stressors. Psychology and Health 14, 1999) 859-868.

[7] Horvath, A. and Greenberg, L. Development and Validation of the Working Alliance Inventory. Journal of Counseling Psychology, 36, 2, (1989) 223-233.

[8] Kamarck, T., Manuck, S., and Jennings, J. Social support reduces cardiovascular reactivity to psychologica challenge: A laboratory model. Psychosomatic Medicine, 52, 1990) 42-58.

[9] Klein, J., Moon, Y., and Picard, R. This Computer Responds to User Frustration: Theory, Design, Results, and Implications. Interacting with Computers, 14, (2002) 119140.

[10] LaVeist, T., Sellers, R., Brown, K., and Nickerson, K. Extreme Social Isolation, Use of Community-Based
Senior Support Services, and Mortality Among African American Women. American J ournal of Community Psychology, 25, 1997) 721-732.

[11] Lester, J. C., Converse, S. A., Kahler, S. E. Barlow, S. T., Stone, B. A., and Bhogal, R. S., The Persona Effect: Affective Impact of Animated Pedagogical Agents, CHI '97, 1997, pp. 359-366.

[12] Liu, K. and Picard, R., Embedded Empathy in Continuous, Interactive Health Assessment, $\mathrm{CHI}$ Workshop on $\mathrm{HCl}$ Challenges in Health Assessment, 2005.

[13] Morkes, J., Kernal, H., and Nass, C., Humor in Task-Oriented Computer-Mediated Communication and Human-Computer Interaction, CHI 98, 1998, pp. 215-216. [14] Mulken, S. v., Andre, E., and Muller, J., An Empirical Study on the Trustworthiness of Life-Like Interface Agents, DFKI, Saarbrucken, Germany, 1999. [15] Reeves, B. and Nass, C., The Media Equation, Cambridge University Press, Cambridge, 1996

[16] Rickenberg, R. and Reeves, B., The Effects of Animated Characters on Anxiety, Task Performance, and Evaluations of User Interfaces, CHI 2000, 2000, pp. 49-56. [17] Watson, D., Clark, L., and Tellegen, A.

Development and validation of brief measures of positive and negative affect: the PANAS scales. J personality and Social Psychology, 54, 1988) 1063-1070.

[18] Wethington, E. and Kessler, R. Perceived Support, Received Support, and Adjustment to Stressful Life Events. Journal of Health and Social Behavior, 27, (1986) 78-89.

[19] Wilson, C. and Turner, D., Companion Animals in Human Health, SAGE Publications, 1997.

[20] Zhang, J., Bernasko, J., Leyborvich, E., Fahs, M., and Hatch, M. Continuous support from labor attendant for primparous women: A meta-analysis. Obstet Gynecol, 88, 1996) $739-44$ 